aural delays, with responsiveness falling off steeply as the virtual location of the stimulus is shifted horizontally. Knowing the preferred sound position of an optic tectum neuron, the authors applied microstimulation in the AGF at a location in its spatial map that either corresponded to the preferred location of the optic tectum neuron or was displaced from it.

What should we expect if the AGF is involved in allocating auditory spatial attention to the preferred location of the stimulated AGF neurons? Neurons in the optic tectum should show some sort of positive attentional modulation if they encoded this stimulated location and show no enhancement or even a suppression if their preference did not match the location. Winkowski and Knudsen found just such effects. The responsiveness of optic tectum neurons was enhanced, but only for sounds very close to the neuron's preferred location. This sharpened the already extremely narrow tuning curves, that is, responses dropped off even more rapidly with appropriate AGF stimulation than without it. Furthermore, responses of neurons with preferred sound locations outside of the stimulated AGF location had suppressed responses.
Let us assume that the owl's allocation of spatial attention to one particular site in its environment is mediated by localized activity in the AGF. According to the new findings ${ }^{1}$, this would modulate the spatial map of environmental sound sources in the optic tectum such that sounds coming from the attended location would create an enhanced representation and other sound sources would be diminished. Not only would this allow the owl to pull out the sounds created by a mouse from other sounds in the environment, but also the attentional sharpening of the tuning would support an enhanced localization of the mouse's exact position.

Such behavioral effects have been documented for stimulations in the $\mathrm{FEF}^{6}$ and the superior colliculus ${ }^{7}$, but for AGF stimulation they await confirmation. Similarly the results of FEF and AGF stimulation leave open the possibility that the attention effects in the V4 and the optic tectum may be indirect. It is possible that the stimulation is interpreted by the organism as a strong, localized saliency signal from the environment that attracts the animal's attention ${ }^{8}$.

The new findings from Winkowski and Knudsen are fascinating for several rea- sons. They document that the allocation of auditory attention in the barn owl and the allocation of visual attention in primates use highly similar control mechanisms. Furthermore, they suggest a tight integration of attentional allocation between modalities, as the AGF controls both visual and auditory gaze. Finally, observing a sharpening of spatial tuning in the optic tectum is important because it has been hypothesized in the visual system ${ }^{9}$ but so far only shown in a preliminary report (T. Womelsdorf \& S.T., Abstr. Soc. Neurosci. 2004).

1. Winkowski \& Knudsen, E.I. Nature (in the press).

2. Moore, T. \& Armstrong, K.M. Nature 421, 370-373 (2003).

3. Sclar, G. \& Freeman, R.D. Exp. Brain Res. 46, $457-$ 461 (1982).

4. McAdams, C.J. \& Maunsell, J.H.R. J. Neurosci. 19 431-441 (1999).

5. Treue, S. \& Martinez-Trujillo, J. C. Nature 399 575-579 (1999).

6. Moore, T. \& Fallah, M. Proc. Natl. Acad. Sci. USA 98 1273-1276 (2001)

7. Müller, J.R., Philiastides, M.G., \& Newsome, W.T Proc. Natl. Acad. Sci. USA 102, 524-529 (2005).

8. Treue, S. \& Martinez-Trujillo, J.C. Curr. Biol. 13, R442-R444 (2003).

9. Moran, J. \& Desimone, R. Science 229, 782-784 (1985).

\title{
Specifying nociceptors
}

The sensory neurons in peripheral sensory ganglia, derived from neural crest precursors, are a highly diverse group of cells. A paper in this issue examines how the emergence of this diversity in the developing neurons is orchestrated. Subgroups of sensory neurons specialize in distinct sensory modalities, such as proprioception, nociception, heat, cold or touch. They differ in size, their projection areas, their degree of myelination, and their expression of receptors, channels and neuropeptides. The bHLH transcription factor Ngn2 is known to specify neural crestderived sensory neurons in general, and a Runt family transcription factor, Runx3, defines the proprioceptive subpopulation. It thus seemed plausible that other Runt family members might be responsible for the differentiation of other sensory neuron subtypes.

Marmigère et al. (page 180) took a close look at Runx1. In chick embryo, Runxl mRNA (blue pseudocolor in the figure) was found in neurons that were positive for TrkA (red immunolabel) but excluded from TrkA-negative cells. (All neurons in the image are co-immunolabeled with the marker Isl1 in green.) The co-localization of Runx1 with TrkA suggests a role for Runx1 in the specification of nociceptor neurons. Developing nociceptors depend on nerve growth factor (NGF) signaling through the TrkA receptor for survival. In a series of experiments overexpressing Runxl and/or

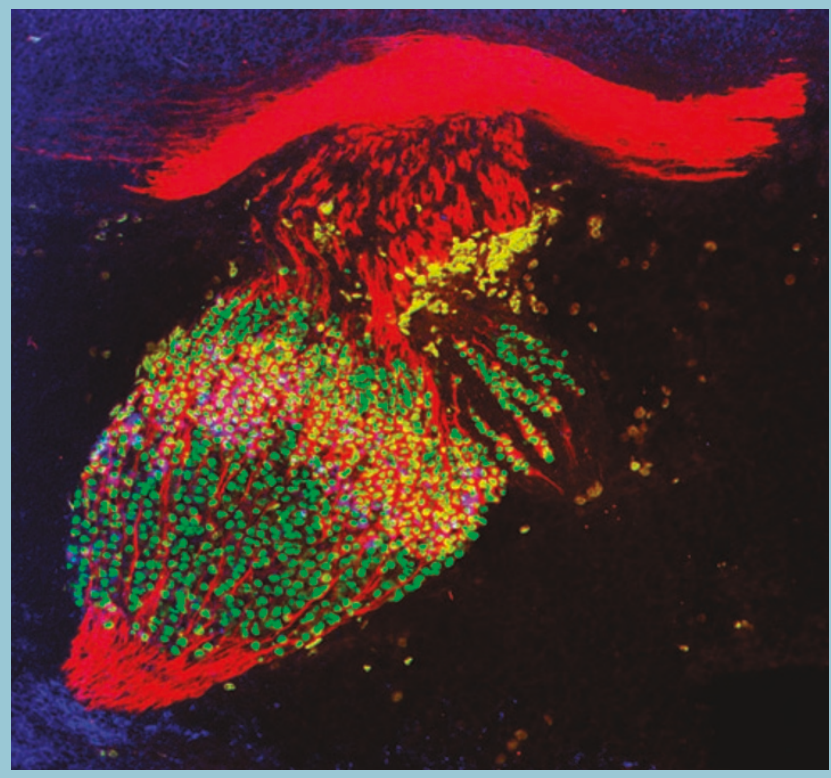
Ngn2 in earlier-stage progenitors, the authors determined that Runx1 acts together with Ngn2 to enable survival and axon growth of young neurons. Runx1 alone supported neither survival nor axon elongation. Suppression of all Runx family transcriptional regulation in ovo with a dominant-negative construct resulted in loss of the nociceptor marker TrkA and death of nearly all transfected neurons, whereas specific knockdown of Runx1 by RNA interference selectively killed the nociceptor subpopulation. The TrkA promoter region contains a putative Runx binding site, so authors presume that Runx1, in collaboration with other transcriptional regulators, directly activates TrkA transcription. Runxl overexpression in progenitors induced precocious expression of TrkA.

Taken together, these results establish Runx1 as a master switch in the development of the neurons that transduce pain signals. Further work will unravel the web downstream of Runx1 and Ngn2, leading to a thorough understanding of nociceptors and hopefully some ideas about how to interfere with pathological pain states.

Annette Markus 